diffusion' disturbances. Satisfactory agreement between theory and experiment was obtained.

Evaluation methods and characterization of their ion-exchange substances were discussed by R. Kunin (Rohm and Haas Co., Philadelphia), W. Bauman (Dow Chemical Co., Midland, Michigan) and W. Utermohlen (Permutit Co., Birmingham, N.J.). Kunin reported methods for capacity determination, which differ considerably for strong and weak cation and anion exchangers. He reviewed both acid and base exchange constants, and it is of interest to note that, for example, 'IRA 400' is a stronger base than sodium hydroxide and will liberate pure sodium hydroxide solution from sodium salts. $\mathrm{He}$ also reported the effects already mentioned of different cross-linking on reaction-rates and on the capacity for large cations, and showed that in the oase of anion exchangers, too, increased cross-linking results in increased specificity and that very considerable variation of the separation factor occurs as a function of the mol-ratio of the absorbed ions. Bauman, after discussing the structure of 'Dowex 50', dwelt particularly on the properties of anions exchangers, such as swelling dependence on cross-linking, chemical stability, titration curves, and exchange constants for a large variety of organic and inorganic anions. The last session of the Conference was devoted to a discussion of engineering factors by $F$. J. Roberts (Dorr Co., Westport, Conn.) and by G. P. Monet (Du Pont Co., Wilmington, Delaware).

The Conference, under the able chairmanship of Dr. R. Kunin, dealt on the average with two papers per session, allowing for a full and free discussion, and this contrasted very favourably with the often very over-crowded discussions of many scientific societies. A feature of the Conference which also deserves recommendation was the arrangement into morning and evening sessions, which left the afternoons free for either private discussions or mental recuperation.

E. GLUECKAUF

1J. Chem. Soc., 1211 (1949).

\section{FERROMAGNETISM AND ANTI-FERROMAGNETISM}

$\mathrm{A}^{\mathrm{A}}$

$\mathrm{N}$ international conference on ferromagnetism and anti-ferromagnetism was held at Grenoble in the first week of July, organised by Prof. L. Néel under the auspices of the Centre National de la Recherche Scientifique with assistance from the Rockefeller Foundation. It was attended by some seventy scientific workers from Europe and America, and a total of about fifty papers were read and discussed in the five days of the conference. In this report it will not be possible to mention all the papers or to give a full account of any of them. References will be given to published papers where these cover more or less the same ground as the reports presented to the conference; the proceedings are to be published in a single volume towards the end of 1950 .

The papers on ferromagnetism reflected the great increase in precise knowledge about Weiss domains and their arrangement which has come about in the past few years, and the consequent possibility of more detailed interpretation of magnetization processes. Those on anti-ferromagnetism illustrated the recent revival of interest in non-metallic magnetic materials, the considerable accumulation of empirical facts and the beginnings of their reduction to order by generalizing theories.

The most direct experimental ev:dence for the size and shape of the domains of a ferromagnetic is obtained by applying a magnetic powder to the surface of the material to reveal the outlines of the domains. This technique was described by Dr. R. M. Bozorth and illustrated by remarkably clear slides of the domain structures observed on various materials ${ }^{1}$. By careful shaping of a single crystal specimen, it is possible to obtain a structure consisting effectively of only two domains, magnetized in opposite directions, with a single boundary wall separating them. Investigations of such specimens were described by Dr. W. Shockley and by Dr. K. H. Stewart, the former showing a film in which the motion of the wall could be followed as the magnetization changed; the way in which small imperfections in the crystal acted as obstacles to the movement of the wall, through the formation of the 'spike domains' predicted theoretically by $\mathrm{Ne}^{2}{ }^{2}$, could be seen clearly. The speed of wall movement in these specimens was found to be controlled by eddy-current effects. In the course of the ensuing discussion, Prof. L. F. Bates showed a very beautiful film of Bitter figures formed on a single crystal of cobalt, which has a particularly simple domain structure; he also showed many pictures of domain boundaries on single crystal surfaces of silicon-iron which provided quantitative proof of the essential correctness of the Néel theory.

The subject of time-effects in changes of magnetization was taken up more generally by Prof. $R$. Becker, who dealt with the relative importance of eddy currents and of the wall inertia effect predicted by Döring ${ }^{3}$. Prof. Néel outlined a new treatment of time-effects, showing that the concept of an internal field changing slowly with time and hence changing the magnetization gave results of wider application than the concept of the magnetization itself changing with time. He distinguished between 'reversible viscosity', which he attributed to changes in magnetocrystalline anisotropy due to the diffusion of atoms of impurities, and 'irreversible viscosity', due to the effects of thermal fluctuations on the movement of domain walls 4 . Experiments on 'irreversible viscosity' were described by J. C. Barbier ${ }^{5}$, and Prof. R. Goldschmidt showed the importance of time-effects in technical applications. The very slow changes in the magnetization of rocks and clays, and the remarkable extent to which they can 'record' past magnetic history, were discussed by Prof. E. Thellier.

Turning from these questions of 'dynamics' to those of 'statics'-the factors controlling the shapes of magnetization curves-the Rayleigh region of the magnetization curve was discussed in the light of Néel's theoretical treatment' by Dr. L. Epelboin and by Dr. L. Lliboutry, the latter considering the effects of small mechanical stresses as well as of small magnetic fields. In the region of high fields, Prof. L. Weil described recent theories of coercive force and their experimental verification; Néel's theory of coercive force in continuous ferromagnetic media ${ }^{2}$ is not easy to check in any detail ; but the theory for ferromagnetics consisting of particles so small as to contain only one domain each (Néel', Stoner and Wohlfarth ${ }^{9}$ ) can be checked in various ways by varving temperature, composition and particle size, and agrees well with experiment. The technical aspects of high-coercivity materials were treated by Dr. K. Hoselitz in a review of progress in the field of permanent magnets. 
Although rapid progress has been made in interpreting magnetization processes in terms of domain structure, there have been no great advances in the more fundamental problems of ferromagnetism, those of the interactions between carriers of magnetic moment leading to a finite spontaneous magnetization in the absence of a field. Prof. E. C. Stoner showed how the 'collective electron' approach ${ }^{10}$, regarding the carriers of magnetic moment as shared between all the atoms of the lattice rather than attached to individual atoms, could give a general account of the behaviour of ferromagnetics more satisfactory in some respects than those of older theories. Prof. R. Forrer extended his previous interpretation ${ }^{11}$ of the saturation moments of alloys in terms of a 'permanent' and a 'supplementary' moment by supposing the latter to be due to an intermittent activation of certain electrons into higher states.

Reports of experimental work on saturation moments and allied questions were given by Prof. W. Sucksmith, who has measured the temperature variation of saturation moment in a cobalt single crystal and in ordered and disordered iron-nickel alloys; Dr. P. 'Taglang, who has determined the moments of several series of 'iso-electronic' alloys; Dr. A. Meyer, who has found the gyromagnetic ratio for iron, njckel and various alloys to be exactly 2 , within the small limits of experimental error; and Prof. L. F. Bates, who outlined the main results of his extensive work on temperature changes during magnetization and showed that they can be related reasonably well to other thermal properties by the thermodynamic theory of Stoner and Rhodes ${ }^{12}$.

Anti-ferromagnetism, which, until recently, has received much less attention than ferromagnetism, is a consequence of negative interactions between carriers of magnetic moment, interactions tending to make the moments of neighbouring carriers point in opposite directions. Nóel ${ }^{13}$ was the first to show that such interactions can lead to just as great ordering of magnetic moments as can the positive interactions characteristic of ferromagnetism, though the resultant magnetic properties are not usually so striking. Recent theoretical developments were outlined at the conference by Prof. J. H. van Vleck. The basic idea is to regard the crystal lattice of the material as made up of two interlocking sub-lattices such that all the neighbours of each atom of one sub-lattice belong to the other sub-lattice; the interaction energy will then be a minimum if all the atomic moments of one sublattice point in the same direction, antiparallel to all the moments of the other sub-lattice, and this ordered arrangement will actually occur if the temperature is below a certain critical value, the Curie point. The quantitative theory, using the concept of a 'molecular field' to represent the interactions, predicts a susceptibility rising slowly to a maximum at the Curie point and thereafter decreasing with increasing temperature. Experimental disagreements with the simple theory indicate clearly that interactions between next nearest neighbours are often of even greater importance than those between nearest neighbours, and make it possible to calculate the ratio of the two types of interaction. The importance of next nearest neighbour interaction can reasonably be interpreted if the interaction is not a simple exchange one but an indirect one of 'superexchange' with an oxygen atom playing the part of intermediary, as suggested by Kramers ${ }^{14}$ and $\mathrm{Ne}^{1{ }^{15}}$. Striking evidence for the division into two sub-lattices below the Curie point has recently been provided by neutron diffraction experiments (Shull ${ }^{16}$ ), showing a periodicity twice as great as the normal lattice spacing.

The experimental study of various anti-ferromagnetic compounds was described by Prof. $\mathbf{H}$. Bizette, Prof. G. Foëx, Dr. F. Trombe, and by Dr. C. Guillaud. Besides determining the Curie temperatures, Prof. Bizette has found the preferred directions of orientation of moments, analogous to the 'easy directions of magnetization', for several crystals.

At very low temperatures many substances exhibit anomalies of specific heat and magnetic behaviour. These were reviewed by Dr. N. Kurti, who showed that further data are required to decide whether the anomalies are evidence of ferromagnetism or of anti-ferromagnetism, and that the ordinary Lorentz dipole interaction field of $\frac{4}{3} \pi I$ is large enough to be of dominating importance in most cases. Similar low-temperature experiments were described by Prof. C. J. Gorter and by Dr. G. C. Garrett.

In the simple anti-ferromagnetics considered so far, the moments of the two sub-lattices are equal and opposite, so that the resultant spontaneous magnetization is zero. Two years ago, Prof. Néel introduced the idea' 15 that many non-metallic 'ferromagnetics' may in reality be imperfectly compensated antiferromagnetics, or 'ferrimagnetics', as he calls them. At the conference he was able to show how fruitful this idea has been in explaining the properties of ferrites and other magnetic oxides. The metallic ions in the ferrite structure $\left(\mathrm{Fe}_{2} \mathrm{O}_{3} \cdot M^{++} \mathrm{O}\right)$ are distributed between two different types of site in the framework of oxygen ions, and Néel supposes that the ions in each type form a sub-lattice in the sense indicated above. Below the Curie point the material will have a spontaneous magnetization equal to the difference between the magnetizations of the two sub-lattices, and in many cases this differs from zero. The theory gives quantitative predictions about the variation of spontaneous magnetization with composition and temperature, the specific heat anomaly at the Curie point and the variation of susceptibility with temperature above the Curie point. Refinements to the theory allow for the effect of orbital as well as spin magnetic moment and for the variation of molecular field with temperature ${ }^{17}$.

Experiments verifying many of these points were reported by Messrs. R. Pauthenet and L. Bochirol ${ }^{18}$, Dr. E. F. Bertaut and Prof. Weil'19, working at Grenoble. More general studies of the properties of ferrites were described by Prof. M. Fallot, Dr. C. Guillaud $^{20}$ and Prof. A. Michel. In this work, as in that of Prof. $\mathrm{R}$. Chevallier on $\alpha-\mathrm{Fe}_{2} \mathrm{O}_{3}$, the difficulties in obtaining pure and homogeneous materials are great, leading at times to confusing results. A new non-metallic magnetic substance was reported by Dr. J. H. van Santen - the material $\mathrm{LaMnO}_{3}-\mathrm{CaMnO}$, with a perovskite structure, has a transformation point at about $300^{\circ} \mathrm{K}$., below which it is ferromagnetic. Since neighbouring manganese atoms are separated by an oxygen atom on their line of centres, this is the first known case of indirect interaction due to pure superexchange; it is also the first case in which indirect interactions have been observed to be positive.

A general review of ferrites was given by Dr. J. L. Snoek, stressing the advances in basic theory but pointing out the problems of high-frequency behaviour still remaining. High-frequency phenomena were also considered in a report by Dr. C. Kittel on gyromagnetic resonance and an account by Mr. F. Roberts of experiments on the radio-frequency analogue of the 
Faraday rotation of the plane of polarization by a magnetized medium.

At the end of the conference, Prof. Bates expressed very happily the gratitude which all the participants, particularly those from other countries, felt towards Prof. Néel and all who helped to organise the conference for the admirable arrangements and the very pleasant atmosphere which prevailed throughout both the formal sessions and the less formal intervals.

${ }^{1}$ Williams, H. J., Bozorth, R. M., and Shockley, W., Phys. Rev., 75, $155(1950)$.

"Néel, L., "Cah. Phys.", 25, 21 (1944).

${ }^{3}$ Döring, w., Z. Naturf., 3a, 373 (1948).

' Néel, L., J. Phys., 11, 49 (1950).

- Barbier, J. C., C.R. Acad. Sci., Paris, 230, 1040 (2950).

'Néel, L., "Cah. Phys.", 12, 1 (1942).

' Néel, L., Ann. Univ. Grenoble, 22, 299 (1946); also Physica, 15, 225 (1949).

${ }^{8}$ Néel, L., C.R. Acad. Sci., Paris, 224, 1550 (1947). - Stoner, E. C., and Wohlfarth, E. P., Phil. Trans. Roy. Soc., A, 240,
599 (1948).

${ }^{10}$ Stoner, E. C., Proc. Roy. Soc., A, 169, 339 (1939). Wohlfarth, E. P., Proc. Roy. Soc., 195, 434 (1949).

${ }^{11}$ Forrer, R., J. Phys., 10, 432 (1939) ; 1, 74 (1940),

${ }^{12}$ Stoner, E. C., and Rhodes, P., Phil. Mag., 62, 481 (1949).

${ }^{13}$ Néel, L.., Ann. de Phys., 17, 64 (1932); 5, 256 (1936).

${ }^{14}$ Kramers, H. A., Physica, 1, 182 (1934).

${ }^{15}$ Néel, L., Ann. de Phys., 3, 137 (1948).

${ }^{16}$ Shull, C. G., and Smart, J. S., Phys. Rev., 76, 1256 (1949).

${ }^{27}$ Néel, L., C.R. Acad. Sci., Paris, 230, 190 (1950).

${ }^{18}$ Pauthenet, R., C.R. Acad. Sci., Paris, 230, 1842 (1950).

${ }^{19}$ Weil, L., Bertaut, F., and Bochirol, L., J. Phys., 11, 208 (1950).

${ }^{20}$ Guillaud, C., J. de Recherches du C.N.R.S.. No. 12 (1950).

\section{STATISTICAL METHODS IN BIOLOGICAL AND CHEMICAL EXPERIMENTS}

I January 1949, the New York Academy of Sciences held a conference on "The Place of Statistical Methods in Biological and Chemical Experimentation"; the fifteen papers there presented are now published* "as a guide to those workers in the fields of biology and chemistry who wish to learn more about the place of statistics in research". The volume naturally does not give a connected account of the methods of statistical science, but it does provide exceedingly interesting illustrations of the many ways in which the statistician can help a research programme. It should be of value to the sceptic requiring to be weaned from his belief that "I don't need statisties in my work", to the teacher in search of examples, and also to the specialist who can appreciate the intrinsic merits of those contributions lying close to his own branch of study.

The first two papers, by G. W. Snedecor and G. M. Cox, are general accounts of the function of statistical analysis and experimental design. Next come several papers descriptive of special statistical techniques, in subjects as diverse as analytical chemistry, nutrition and immunology. Four papers on biological assay and three on studies of human populations complete the collection. All the authors have something worth saying, and all present their topics with admirable clarity; one or two need special mention.

Many readers will be interested to see $F$. W. Wilcoxon's survey of the approximate methods of analysis that he has done so much to develop. C. V.

- Annals of the New York Academy of Sciences. Vol. 52, Art. 6 : The Place of Statistical Methods in Biological and Chemical Experimentation, By Edwin J. de Beer, Lloyd C. Miller and 14 other Authors. Pp. 789-942. (New York : New York Academy of Sciences,
1950.) 2.25 dollars.
Winder contributes a valuable account of certain applications of statistics in pharmacology, in the course of which he discusses the choice of a metameter for analysis. L. C. Miller compares different techniques for the analysis of assays using quantal responses, making useful comment on the validity and utility of each. D. Mainland and D. D. Reid describe some of the statistical problems of research in clinical medicine, with special reference to recent progress in this important and difficult field.

D. J. FInNeY

\section{THE TSETSE FLY IN EAST AFRICA}

THE report of the East African Tsetse and Trypanosomiasis Organisation for 1949* shows that an integrated attack is being made upon this many-sided problem-research and reclamation are being closely co-ordinated, the medical, veterinary and agricultural aspects are being treated as different facets of a single problem, and progress in East Africa is being correlated at each step with advances made in other parts of the continent.

As the director, Dr. H. M. O. Lester, points out, the broad aim of the Organisation is the assistance of development in East Africa; and the reorganisation of research which is in progress and, in particular, the closer liaison between tsetse research and trypanosomiasis research, are clearly having a stimulating effect. Detailed work with improved technique is in progress to define exactly the habitats of the different species of tsetse fly so as to make possible the eradication of the tsetse with the minimum interference with vegetation.

There are many questions in tsetse biology which must be answered before land-utilization policies can be formulated. It is already clear that the eradication of tsetse fly from any area has little value unless the land that has been freed from this pest is required for some specific purpose. Until more effective methods of applying insecticides and cheaper methods of clearance are developed, eradication and resettlement will only be economic where there is sufficient pressure of population for the organisation of large-scale undertakings. 'The effect of 'fire exclusion' in exterminating Glossina swynnertoni has been confirmed. As in Southern Rhodesia, it has been proved in an experimental fenced area of 560 square miles that the destruction of game animals leads to a very great reduction in the numbers of $G$. morsitans and the virtual collapse of $G$. swynnertoni.

On the veterinary side, extensive trials have been carried out on the prophylactic and curative value of the drug 'Antrycide'. A cautious attitude was adopted from the outset by the research organisation, and this attitude appears to have been justified. The drug has remarkable curative properties against veterinary trypanosomiasis, although severe toxic reactions have occurred and will require further study before its use on a large scale can be recommended. As a prophylactic the drug has proved disappointing. It is liable to cause prolonged incubation of the disease and so to mask infection; and acquired resistance is so readily developed by $T$. congolense and $T$. vivax that it appears difficult, if not impossible, to use the drug for large-scale prophylaxis in East Africa.

- East African High Commission: East African Tsetse and Trypanosomiasis Research and Reclamation Organisation. Annual Report, 1949. By Dr. H. M. O. Lester. Pp. 26. (Nairobi : East
African High Commission, 1950.) 